\title{
The deflationary effect of oil prices in the
} euro area

\author{
César Castro*1 ${ }^{* 1}$ Miguel Jerez ${ }^{\dagger 2}$ and Andrés Barge-Gil ${ }^{\ddagger 2}$ \\ ${ }^{1}$ Department of Economics, University of Salamanca, Spain. \\ ${ }^{2}$ Department of Economic Analysis II, Universidad Complutense de Madrid, Spain
}

February 22, 2016

\begin{abstract}
The inflationary effect of oil price has been widely examined by academic literature. Nowadays, the main concern in the euro area (E.A.) is its deflationary effect. In this paper we propose a method to evaluate the effect of oil price changes on inflation as well as an indicator of inflation adjusted for the short-term effect of oil prices, which is aimed to assess the risk of deflation in real time. We illustrate the practical applications of these tools by predicting the evolution of inflation in the E.A., conditional to different scenarios of oil price deflation. Our main finding is that no deflationary scenario for oil prices results in a negative inflation rate forecast for October 2016, despite oil price variation accounting for $25 \%$ of the variance of changes in inflation.
\end{abstract}

Keywords. Inflation, Deflation, Oil price, Euro area, Forecasts.

\section{Introduction}

The relevance of oil prices as a source of variations in prices is established since the 1970s. However, in the last two decades several works have documented that this relevance has decreased. Hooker 2002 finds no significant impact of oil prices changes on U.S. inflation, excluding energy products. DeGregorio et al. 2007] document an important reduction in the contribution of oil prices changes on consumer prices, providing evidence for a sample of 34 countries. Blanchard and Galí 2010 find that the inflationary impact of crude costs decreased since mid 1980s. Kilian 2008a b] states that the effect of exogenous oil prices shocks

\footnotetext{
*Corresponding author. E-mail: ccastrorozo@gmail.com

${ }^{\dagger}$ E-mail: mjerezme@ucm.es

‡E-mail: abarge@ccee.ucm.es
} 
on inflation in G7 countries is quite small and highlights its heterogeneity across countries. Álvarez et al. 2011] find that the contribution of oil price changes is limited, but still constitutes a major driver of inflation variability in Spain and the euro area, mainly through direct effects.

Several reasons have been proposed to explain this loss of relevance (e.g., DeGregorio et al. 2007, Blanchard and Galí [2010]): higher energy efficiency of production processes, relevance of globalization or changes in the conduct of monetary policy.

The emphasis of academic analyses also changed. Previous studies traditionally focused in assessing the inflationary effect of the increases in oil price. However, the main concern in the recent months is the risk of a deflation spiral unchained by oil prices reductions.

The main contributions of this paper are: (i) a method to assess the effects of oil prices changes in inflation under different oil price scenarios and (ii) a modelbased indicator of inflation adjusted for the short-term effect of oil prices, being this indicator a potentially useful tool to track in real time the risk of deflation. We illustrate the practical application of these tools by means of a simulation analysis of the risk of deflation in the euro area (E.A.). ${ }^{1}$

To this end, we first fit a time series model relating the annual variation rates of inflation and oil price. Its dynamic structure implies that the price of crude oil in any given month affects consumer prices in the same month and the month after, with no feedback in the opposite direction of Granger causality. We provide several justifications for this assumption, as well as a Granger-causality test for the E.A.

With this model we: (a) compute twelve-months ahead forecast for inflation in the E.A., conditional to different scenarios of oil price deflation and (b) estimate which part of the recent evolution of consumer prices can be attributed to changes in oil prices. This analysis incorporates two novelties: an interpolation method to compute forecasts conditional to any predetermined terminal value using a fixed-interval smoother (see Anderson and Moore 1979]), and the procedure developed by Casals et al. 2010,, which computes the contribution of each input to the output for any model in transfer function form.

The main results of this analysis are: (a) negative inflation is not expected for the twelve-months-ahead forecasts in any of the three scenarios, (b) the short-time effect of oil on consumer prices is important, as it accounts for $25 \%$ of the variance of changes in inflation so, (c) a spiral of deflation/economic contraction could finally happen if a long period of anemic inflation/deflation affects the consumer expectations and, through them, the economic activity.

The paper is organized as follows. Section 2 discusses the methodological foundations, describes the data and provides a preliminary exploration of their dynamic properties. Section 3 describes the model-building process and Section 4 discusses the empirical results. Finally, Section 5 provides some concluding remarks.

\footnotetext{
${ }^{1}$ E.A. refers to the respective country compositions at a specific point in time: E.A.112000, E.A.12-2006, E.A.13-2007, E.A.15-2008, E.A.16-2010, E.A.17-2013, E.A.18-2014, and E.A.19-2015. Euro area is the official name for the Eurozone
} 


\section{Methodology}

\subsection{Methodological issues}

Our analysis concentrates in the effect of oil prices over inflation in the E.A. An important issue when developing this analysis is to consider the possibility of a feedback relationship, with inflation explaining oil prices.

There is a widespread agreement in the current literature that oil price should be considered endogenous with respect to macroeconomic aggregates, in particular with respect to U.S. GDP growth (see, e.g., Barsky and Kilian 2004; Kilian 2008c; Hamilton 2009; Kilian 2014). This idea is based in the weight of U.S. GDP growth on the global demand, including oil demand, and hence on oil prices.

On the other hand, the Granger (G- causality) test has often been used to test whether U.S. inflation help in predicting oil price changes. G-causality is usually not found after 1975 (Hooker 1996, Gillman and Nakov 2009, Alquist et al. 2013). ${ }^{2}$

The E.A. shows three important differences with U.S.: its lack of internal oil production, its smaller economic size 3 and its lower influence through monetary policy. Accordingly, we will first assess whether E.A., with $13 \%$ of global oil consumption is large enough to determine oil prices. In comparison, the consumption in U.S. has been 23\%. To this end, in the next Sub-section we test for linear G-causality, finding no significant influence of E.A. inflation on oil prices.

Building on this negative result, we use in our analysis a transfer function (TF) specification (Box et al. 1994]), relating oil price (cause) to E.A. inflation (effect) instead of the vector autoregressive (VAR) framework model (e.g., Hamilton [1983]; Jiménez-Rodríguez and Sánchez 2005]; Kilian 2009; Blanchard and Galí [2010]).

The main reason for this choice is that, if no relation exists between lagged inflation and current oil price, then the bidirectional VAR representation looses its main advantage when compared to the unidirectional TF model. On the other hand, the TF model has two compelling advantages for our analysis.

First, the TF is a structural model, allowing one to attribute the contemporary correlation to a specific causal direction, while the reduced-form VAR model captures this correlation in a non-causal way. This is specially important in our analysis because the contemporaneous correlation between oil price changes and inflation: (a) has been shown to be unidirectional (Kilian and Vega 2011]), (b) is much stronger than the lagged ones, so it (c) has a strong contribution to the point forecasts and fitted values for inflation employed in our

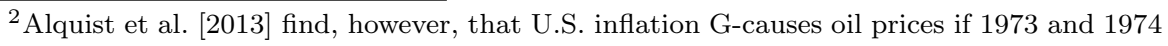
are included in the analysis

${ }^{3}$ In terms of its share of global GDP in PPP in 2014, the euro area is the world's thirdlargest economy (12.2\%), after the United States (15.9\%) and China (16.6\%).

${ }^{4}$ This percentage has been obtained as the mean participation between 1996 and 2014 of the oil consumption of 11 European countries (Austria, Belgium, Finland, France, Germany, Greece, Ireland, Italy, Netherlands, Portugal and Spain) in the total world. These data come from the British Petroleum Statistical Review of World Energy 2014 http://www.bp.com. 
analysis.

Second, inflation displays a strong seasonal fluctuation which is easier to capture in the ARIMA model for the errors of a TF than in a VAR framework.

\subsection{The data}

In this Sub-section, we provide a exploratory analysis of the data that we will model later.

We will denote by $P_{t}^{E A}$ the Harmonized Index of Consumer Price (HICP), by $O_{t}^{E U R}$ the nominal price of Brent in $€$ per barrel, by $O_{t}^{U S \$}$ the price of Brent in US $\$$ and by $E R_{t}$ the exchange rate $€ / \mathrm{US} \$ 5^{5}$ In all cases the observation frequency is monthly and the sampling period runs from January 1996 to December 2015.

Building on this data we computed the annual percent variation rates, defined by:

$$
r^{12}\left(x_{t}\right)=\left(\frac{x_{t}}{x_{t-12}}-1\right) \times 100
$$

Hence these basic variables often appear transformed in annual percent rates: $r^{12}\left(O_{t}^{E U R}\right)$ for annual percent change in oil prices, and $r^{12}\left(P_{t}^{E A}\right)$ for inflation in the E.A.

The profile of these series is shown in Figure 1. The second and third panels allows us to identify several oil price periods: First, the negative shock started in 1997, caused mainly by falls in oil market-specific demand following Asian crisis of 1997-1998. This effect was accompanied by positive rebounds started in late 1998 that reaches its maximum in February 2000. Second, several sustained positive rates between late 2002 and early 2008, driven by global aggregate demand originates in a stronger economic growth, especially in Asian economies. This effect was reversed by a sharp drop in prices associated with the global crisis of 2007-2009. This effect was also accompanied by positive rebounds started in 2009 that reaches its maximum in December 2009. Finally, there has been a sustained fall in prices since late 2009, associated with a strong global supply and a weak global demand 6 The correlation between $r^{12}\left(O_{t}^{E U R}\right)$ and $r^{12}\left(O_{t}^{U S}\right)$ is 0.952 , suggesting that the exchange rate plays a minor role in determining the oil price in Euros. This preliminary result is further confirmated by the analysis in section 3.2., models (4)-(5).

As expected, these series display changes in the mean, so their stationary transformation would be a first-order difference 7 Accordingly, the resulting

\footnotetext{
${ }^{5}$ The HICP data come from http://ec.europa.eu/eurostat). We consider the oil price in U.S. dollar downloaded from the U.S. Energy Information Administration (EIA) web page http://www.eia.gov and use the monthly average exchange rate published by the OECD http://www.stats.oecd.org to calculate the equivalent value in euros.

${ }^{\circ}$ For details see e.g., Kilian 2009.

${ }^{7}$ In our opinion, $O_{t}^{E U \bar{R}}$ could either be I(1) or display a weak seasonality which is buried by its high volatility. However, we work with the same transformation in both variables because the underlying assumption is that oil prices affect consumer prices, so annual inflation must be affected by the annual growth rate of oil prices, no matter that the minimum-order stationary
} 

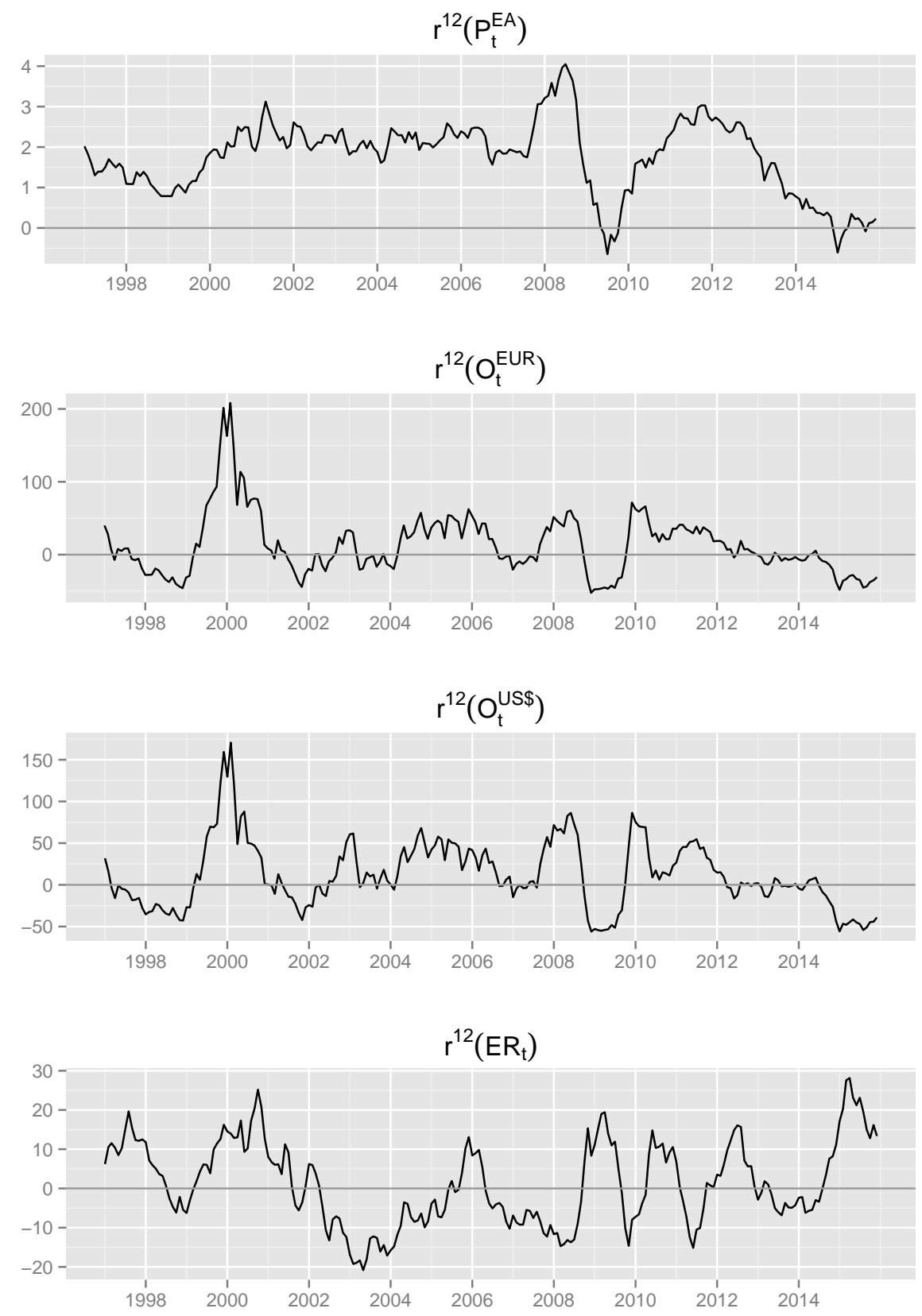

Figure 1: Annual percent changes for inflation in the euro area $r^{12}\left(P_{t}^{E A}\right)$, Brent price per barrel in euros $r^{12}\left(O_{t}^{E U R}\right)$ and US dollars $r^{12}\left(O_{t}^{U S \$}\right)$, and exchange rate $€ /$ US $\$, r^{12}\left(E R_{t}\right)$. Source: Eurostat, EIA and OECD. 
variables can then be interpreted as the monthly acceleration in the inflation rate and annual rate of growth of oil prices, respectively. Figure 2 shows the profile of these series 8 .

Table 1 displays some descriptive statistics for the stationary transform. The $p$-values in the table show that 1st-differenced transformation of annual rates assure the stationarity. Note that the volatility of $\nabla r^{12}\left(O_{t}^{E U R}\right)$ and $\nabla r^{12}\left(O_{t}^{U S \$}\right)$ is approximately 65 times higher than that of the $\nabla r^{12}\left(P_{t}^{E A}\right)$.

\begin{tabular}{rcccc}
\hline & $\nabla r^{12}\left(P_{t}^{E A}\right)$ & $\nabla r^{12}\left(O_{t}^{E U R}\right)$ & $\nabla r^{12}\left(O_{t}^{U S \$}\right)$ & $\nabla r^{12}\left(E R_{t}\right)$ \\
\hline Mean & -0.01 & -0.31 & -0.31 & 0.03 \\
Std. Dev. & 0.24 & 15.82 & 15.32 & 3.57 \\
Minimum & -1.05 & -79.75 & -70.89 & -8.78 \\
Maximum & 0.74 & 57.63 & 49.42 & 10.25 \\
$p$-value ADF & 0.01 & 0.01 & 0.01 & 0.01 \\
$p$-value KPSS & 0.10 & 0.10 & 0.10 & 0.10 \\
\hline
\end{tabular}

Table 1: Descriptive statistics for the stationary series for inflation in the euro area $\nabla r^{12}\left(P_{t}^{E A}\right)$, Brent price per barrel in euros $\nabla r^{12}\left(O_{t}^{E U R}\right)$ and dollars $\nabla r^{12}\left(O_{t}^{U S \$}\right)$ and exchange rates $€ / \mathrm{US} \$ \nabla r^{12}\left(E R_{t}\right)$.

\subsection{Granger causality test}

We will now perform the standard G-causality test (see Granger [1969]) for a fitted VAR model to assess whether (a) past values of oil prices changes in $€$ help in predicting current inflation changes in the E.A. and/or b) there exists the corresponding inverse G-causality effect. If causal effects in Granger sense operate in both directions, then both variables would be endogenous and a vector autoregressive (VAR) model would be needed to obtain consistent estimates for the corresponding dynamic feedback structure.

The G-causality test is implemented by the regressions:

$$
\begin{gathered}
\nabla r^{12}\left(P_{t}^{E A}\right)=c^{1}+\alpha_{1} \nabla r^{12}\left(P_{t-1}^{E A}\right)+\beta_{1} \nabla r^{12}\left(O_{t-1}^{E U R}\right)+\mu_{t}^{1} \\
\nabla r^{12}\left(O_{t}^{E U R}\right)=c^{2}+\alpha_{2} \nabla r^{12}\left(O_{t-1}^{E U R}\right)+\beta_{2} \nabla r^{12}\left(P_{t-1}^{E A}\right)+\mu_{t}^{2}
\end{gathered}
$$

Table 2 shows the $p$-values for each $F$-test with the lag order $p=1$ chosen according to the Schwartz Information Criterion (SIC). Due to the differencing used to induce stationarity on the series, that is, the monthly change in annual rates, the lag order $p=1$ implies effects longer than a year. We do not find evidence of G-causality from E.A. inflation to oil prices, although we find very strong evidence (at $1 \%$ significance) that $\nabla r^{12}\left(O_{t}^{E U R}\right)$ Granger-cause $\nabla r^{12}\left(P_{t}^{E A}\right)$. The replication of this exercise for the U.S. (results available upon request to the authors), shows a p-value of 0.0877 when analyzing G-causality

transform for each series can be different.

${ }^{8} \nabla=(1-L)$ is the difference operator, such that $\nabla \omega_{t}=\omega_{t}-\omega_{t-1}$. 

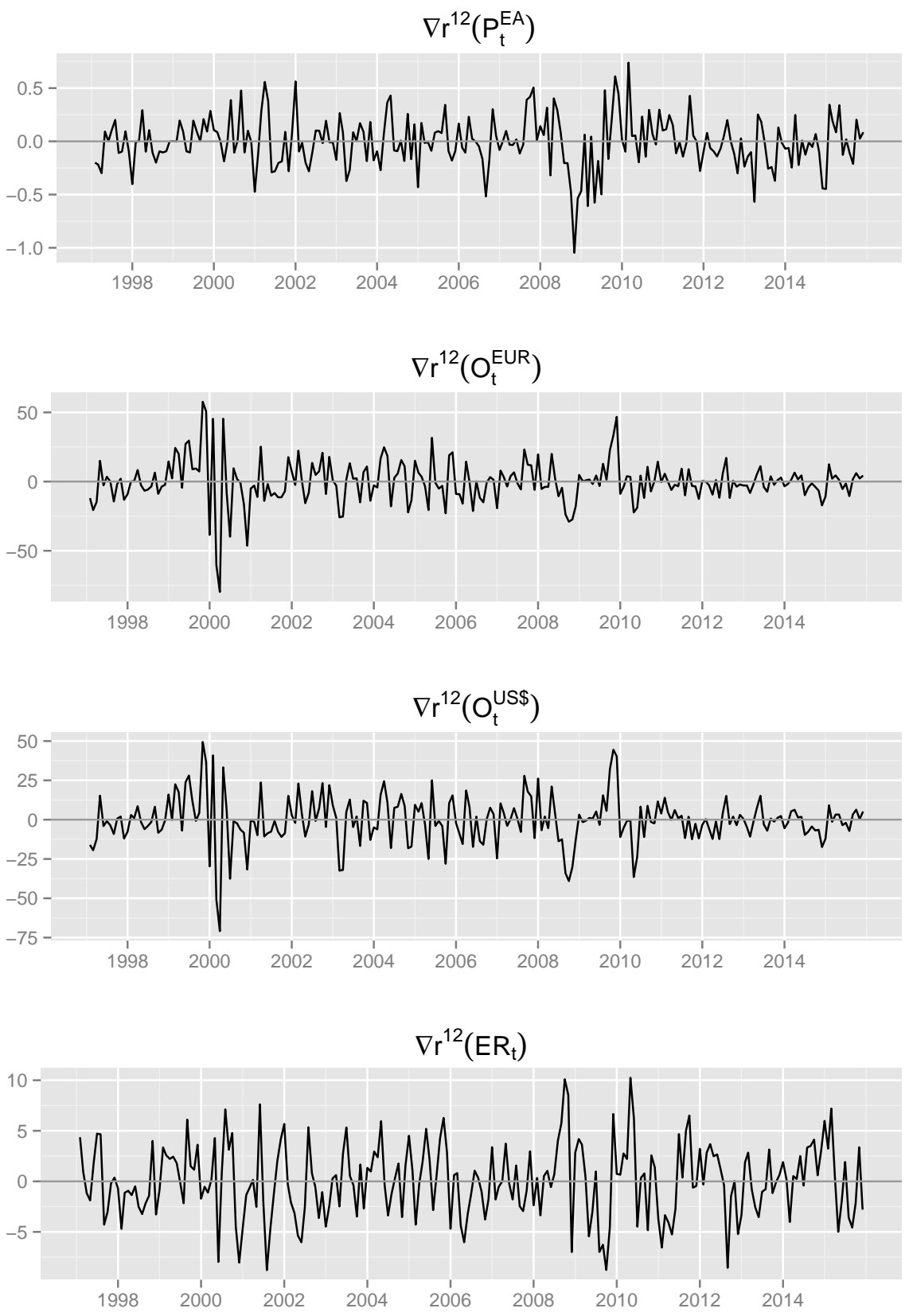

Figure 2: Stationary series for inflation in the euro area $\nabla r^{12}\left(P_{t}^{E A}\right)$, Brent price per barrel in euros $\nabla r^{12}\left(O_{t}^{E U R}\right)$ and dollars $\nabla r^{12}\left(O_{t}^{U S \$}\right)$ and exchange rate $€ /$ US $\$, \nabla r^{12}\left(E R_{t}\right)$. Source: Eurostat, EIA and OECD. 
from U.S. inflation to oil prices in dollars. This results supports the view that the relationship between oil prices and inflation is very different between E.A. and U.S. and advise against using our methodology to analyze the relationship between U.S. inflation and oil prices.

Table 2: $p$-values for linear G-Causality test. The test is calculated with a VAR model. The lag order has been selected according to the Schwarz Information criterion (SIC). One/two/three asterisks denote significance at the $10 \%, 5 \%$ and $1 \%$ levels, respectively.

\begin{tabular}{clll}
\hline $\operatorname{lag}$ & $\nabla r^{12}\left(P_{t}^{E A}\right) \nrightarrow \nabla r^{12}\left(O_{t}^{E U R}\right)$ & $\nabla r^{12}\left(O_{t}^{E U R}\right) \nrightarrow \nabla r^{12}\left(P_{t}^{E A}\right)$ \\
\hline 1 & 0.359326 & 0.000002 & $* * *$ \\
\hline
\end{tabular}

\section{Models}

\subsection{ARIMA Models}

The main purpose of our analysis consists in modelling the relationship between the inflation rate in the euro area $\nabla r^{12}\left(P_{t}^{E A}\right)$ and the annual percent growth of Brent prices in $€, \nabla r^{12}\left(O_{t}^{E U R}\right)$. The basic shortcoming of this approach is that the world market is quoted in US\$, so the latter variable confounds the effects of oil price changes with those due to fluctuations in the exchange rates.

To solve this issue we will take into account that $O_{t}^{E U R}=O_{t}^{U S \$} \times E R_{t}$, where $O_{t}^{U S \$}$ denotes the nominal prices of Brent in US\$ and $E R_{t}$ denotes the exchange rate $(€ / \mathrm{US} \$)$ at month $\mathrm{t}$, so that the effect of oil prices changes in US\$ are separated from the effect of exchange rate variations.

To accomplish the analysis we start by fitting ARIMA models to the annual rates of inflation and Brent prices in $€$ and US\$. The main estimation and diagnostic results are summarized in Table 3. These models are used for different purposes, including forecasting and prewhitening, see Box et al. 1994.

Note that the residual standard deviations of the annual rates of Brent prices is approximately 66 times higher than that of inflation in the E.A. This is a critical feature of these variables which explains, e.g., that: (a) the $€ /$ US $\$$ exchange rate is irrelevant to our analysis and (b) the coefficients relating changes in oil prices with changes in inflation are small in absolute terms.

\subsection{Transfer function models}

We start by modelling the relationship between the inflation rate in the euro area $r^{12}\left(P_{t}^{E A}\right)$ and the annual percent growth of Brent prices in $€, r^{12}\left(O_{t}^{E U R}\right)$. The relationship has been specified by (a) prewhitening both the input and output series using the ARIMA model for the input (see table 3), and then (b) computing the cross-correlation function between the prewhitening values of both variables, which is shown in figure 3 . 
Table 3: ARIMA modelling results corresponding to $\operatorname{ARIMA}(3,1,0) \times(0,0,1)_{12}$ process for $r^{12}\left(x_{t}\right)$. The figures in parentheses are the standard errors of corresponding parameters.

\begin{tabular}{lccc}
\hline Variable & $\nabla r^{12}\left(O_{t}^{E U R}\right)$ & $\nabla r^{12}\left(O_{t}^{U S \$}\right)$ & $\nabla r^{12}\left(P_{t}^{E A}\right)$ \\
\hline$\phi_{1}$ & 0.048 & 0.133 & 0.208 \\
& $(0.065)$ & $(0.066)$ & $(0.065)$ \\
$\phi_{2}$ & -0.088 & -0.104 & \\
& $(0.065)$ & $(0.066)$ & \\
$\phi_{3}$ & 0.222 & 0.165 & \\
& $(0.065)$ & $(0.066)$ & \\
$\Theta_{1}$ & -0.535 & -0.547 & -0.546 \\
& $(0.06)$ & $(0.06)$ & $(0.053)$ \\
$\sigma_{a}$ & 13.557 & 13.005 & 0.203 \\
$Q(39)(p$-value $)$ & $43.247(0.16)$ & $49.73(0.051)$ & $41.096(0.296)$ \\
\hline
\end{tabular}

The profile of the cross-correlations suggests that inflation is positively correlated with the change in Brent prices in the same month and the month before. In the inverse direction of G-causality (i.e., with current inflation affecting future changes in Brent prices) there is not any significant negative correlation. Accordingly, we confirm our previous findings that G-causality goes from changes in Brent prices to inflation.

On the basis of this statistical analysis, our tentative specification was: (a) a relation term where $r^{12}\left(P_{t}^{E A}\right)$ is a function of $r^{12}\left(O_{t}^{E U R}\right)$ and $r^{12}\left(O_{t-1}^{E U R}\right)$, combined with (b) an $\operatorname{ARIMA}(0,1,1) \times(0,0,1)_{12}$ model for the error, which coincides with the ARIMA specification chosen for the output, see table 3 . This specification provides the following estimation results?

$$
\begin{gathered}
\left.r^{12}\left(P_{t}^{E A}\right)=\underset{\left(9 \times 10^{-4}\right)}{(0.0053}+\underset{\left(9 \times 10^{-4}\right)}{0.0044} L\right) r^{12}\left(O_{t}^{E U R}\right)+\hat{N}_{t}^{P} \\
\left(1-\underset{(0.066)}{0.1389 L)} \nabla \hat{N}_{t}^{P}=\underset{(0.055)}{(1-0.4517} L^{12}\right) \hat{a}_{t}^{P} \\
\hat{\sigma}_{P}=0.184 \quad \log -l i k=60.819
\end{gathered}
$$

Model (1) confounds the effects of oil price changes with those due to fluctuations in the exchange rates. To solve this issue we should take into account that $O_{t}^{E U R}=O_{t}^{U S \$} \times E R_{t}$, so the input variable in model (1) can be decomposed in the following way:

$$
r^{12}\left(O_{t}^{E U R}\right) \simeq r^{12}\left(O_{t}^{U S \$}\right)+r^{12}\left(E R_{t}\right)
$$

and this decomposition suggests building a new model relating the inflation rate in the euro area, $r^{12}\left(P_{t}^{E A}\right)$, with the annual growth of Brent prices in US\$

\footnotetext{
${ }^{9}$ In these equations the letter $L$ denotes the backshift operator, such that for any sequence $\omega_{t}: L^{i} \omega_{t}=\omega_{t-i}, i=0, \pm 1, \pm 2, \ldots, I$.
} 


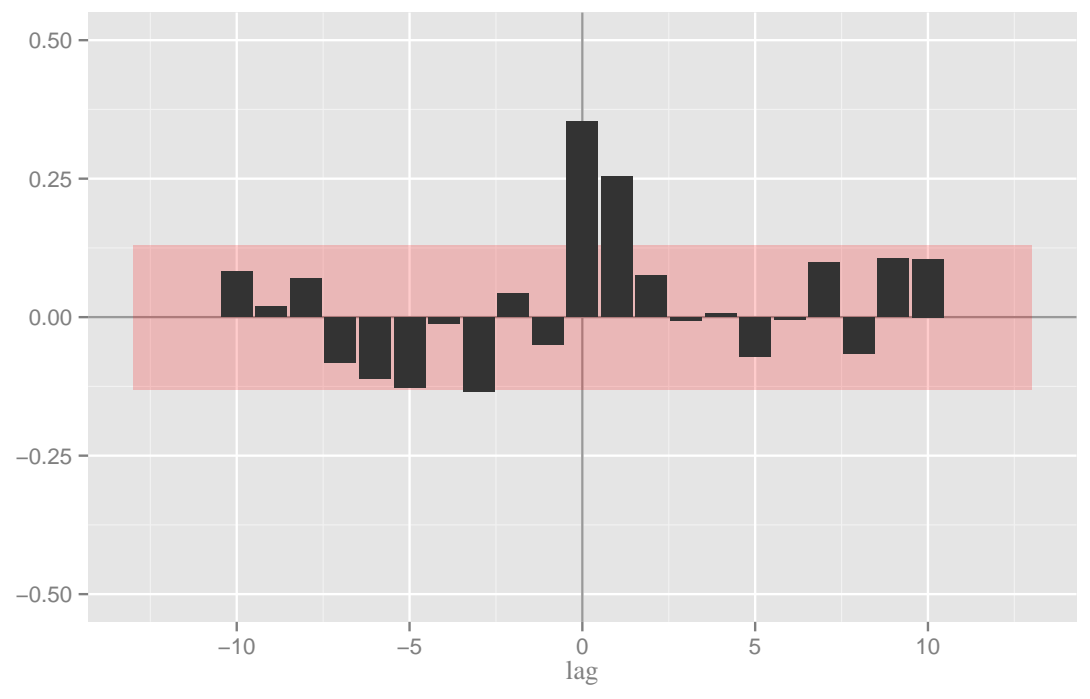

Figure 3: Cross correlations between the prewhitened series of inflation in the euro area, $\nabla r^{12}\left(P_{t}^{E A}\right)$ and the lagged annual variation rate of Brent prices in euros. The shaded area is approximate $5 \%$ significance limits for each individual correlation. Note that negative lags are actually leads for $\nabla r^{12}\left(O_{t}^{E U R}\right)$.

$r^{12}\left(O_{t}^{U S \$}\right)$ and the annual growth of the exchange rate, $r^{12}\left(E R_{t}\right)$. The main estimation results for this specification are the following:

$$
\begin{gathered}
\left.r^{12}\left(P_{t}^{E A}\right)=\underset{\left(9 \times 10^{-4}\right)}{(0.0057}+\underset{\left(9 \times 10^{-4}\right)}{0.0047} L\right) r^{12}\left(O_{t}^{U S \$}\right) \\
+\underset{(0.004)}{(0.0011}+\underset{(0.004)}{0.0021 L} L) r^{12}\left(E R_{t}\right)+\hat{N}_{t} \\
\left(1 \underset{(0.067)}{0.1351 L) \nabla \hat{N}_{t}}=\underset{(0.055)}{(1-0.4424} L^{12}\right) \hat{a}_{t} \\
\hat{\sigma}_{P}=0.182 \quad \log -l i k=63.348
\end{gathered}
$$

where the parameters associated to the exchange rate are non-significant. This result justifies the following final model:

$$
\begin{gathered}
\left.r^{12}\left(P_{t}^{E A}\right)=\underset{\left(9 \times 10^{-4}\right)}{(0.0056}+\underset{\left(9 \times 10^{-4}\right)}{0.0046} L\right) r^{12}\left(O_{t}^{U S \$}\right)+\hat{N}_{t}^{P} \\
\left(1-\underset{(0.066)}{0.1351 L)} \nabla \hat{N}_{t}^{P}=\underset{(0.055)}{(1-0.4462} L^{12}\right) \hat{a}_{t}^{P} \\
\hat{\sigma}_{P}=0.182 \quad \log -l i k=63.166
\end{gathered}
$$


where the likelihood value is: (a) almost identical to the one achieved in model (4)-(5), so both models can be considered statistically equivalent and (b) larger than that of model (1)-(2), implying that the final model would be preferred to models (1)-(2) and (4)-(5) according to any Information Criterion 10

Model (6)-(7) has been submitted to a standard diagnostic testing process which includes:

1. computing the sample cross-correlation function of the model residuals against the prewhitened values of the input, see Figure 4. first panel, which shows no evidence of additional cross-correlation structure,

2. computing the sample cross-correlation function of the same residuals against the prewhitened values of the $€ /$ US $\$$ exchange rate, see Figure 4. second panel, to assure that inflation in the E.A. does not display any significant reaction to changes in the exchange rate, and

3. overfitting experiments, in which we arbitrarily augmented the lag structure of model (6)-(7); the corresponding parameters were non-significant in all cases.

\section{Results}

\subsection{Assessing the likelihood of deflation}

As the results in previous section show, changes in oil pricing have a significant effect on inflation. Accordingly, this variable is relevant to compute short-term inflation forecasts and, in general, to determine the monetary policy. As oil prices are highly volatile and, therefore, difficult to predict stricto sensu, it is reasonable to compute inflation forecasts conditional to a variety of oil price scenarios.

Accordingly, we will now compute the inflation paths corresponding to different scenarios for oil prices, using the model (6)-(7). To this end, first formulate the basic assumptions described in Table 4 .

After setting these assumptions, we need to compute the most likely path for oil prices to reach the assumed annual variation rates. To this end, we created three new variables by joining: (a) the past history of $r^{12}\left(O_{t}^{U S \$}\right)$ until December 2015, (b) eleven missing values corresponding to the months between January 2016 and November 2016, and (c) the value of $r^{12}\left(O_{t}^{U S \$}\right)$ corresponding to December 2016 according to each scenario. The missing values were then interpolated by processing this sample with a fixed-interval smoother, see Anderson and Moore [1979], assuming that the data generating process is the

\footnotetext{
${ }^{10}$ In all the transfer function models, we identified some outliers related with the sharp fall in oil prices started at the end of 2008. Although these outliers are statistically significant, the models reported in the main text do not include the corresponding intervention terms because they do not affect significantly the results of the analysis. We provide them in the Appendix.
} 

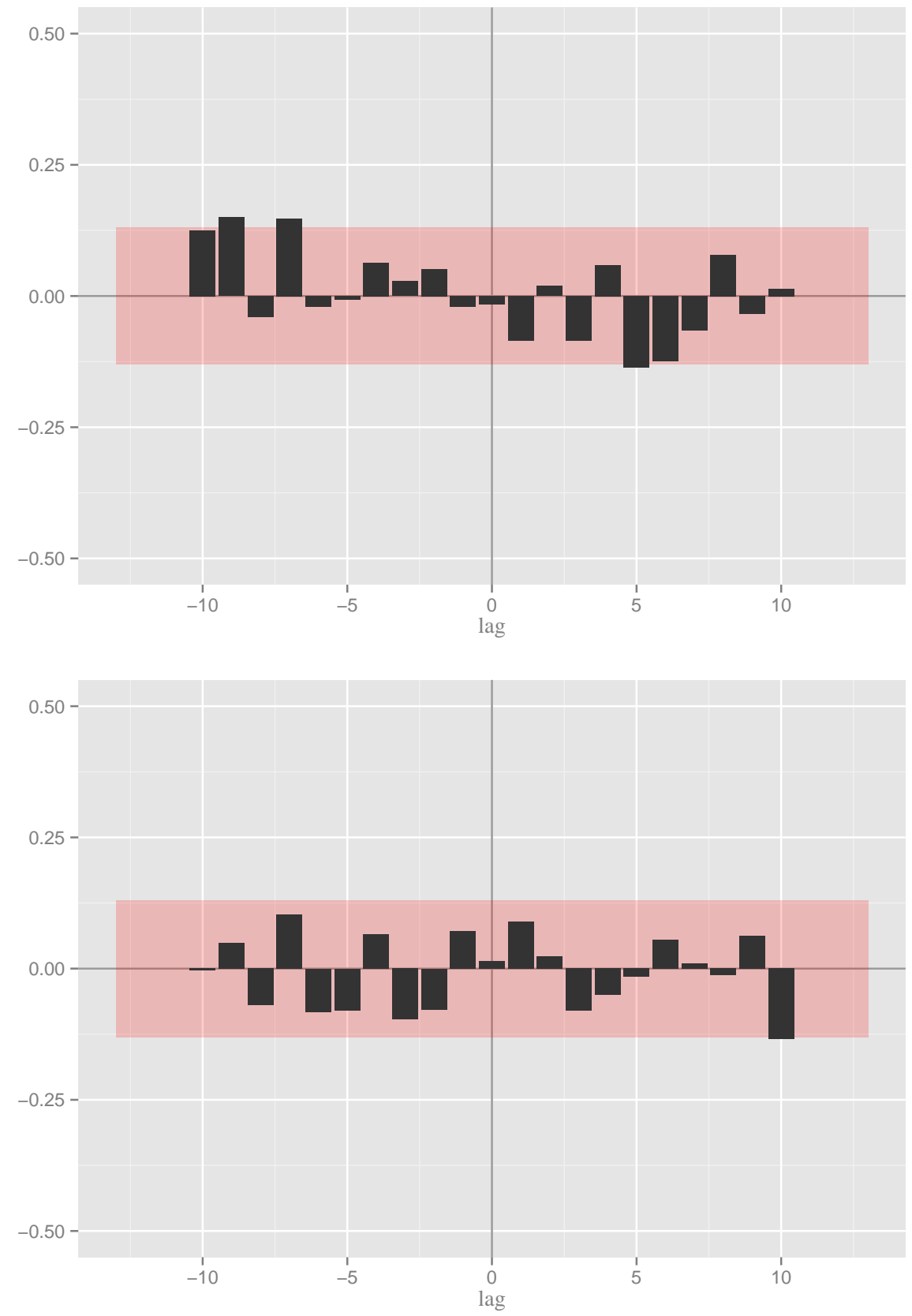

Figure 4: Cross correlations between: (a) the prewhitened annual variation rate of Brent prices and the lagged residuals of model (first panel), and (b) the same residuals and the prewhithened $€ /$ US $\$$ exchange rate (second panel). 


\begin{tabular}{lcc}
\hline Scenario & Assumed price & Annual variation rate \\
\hline Stable & 37.97 & $0 \%$ \\
Pessimistic & 30.00 & $-21.0 \%$ \\
Extreme & 20.00 & $-47.3 \%$ \\
\hline
\end{tabular}

Table 4: Scenarios for Brent prices (US\$/Barrel) in December 2016 versus December 2015. The assumptions for pessimistic and extreme scenario consider that surplus of oil will persist in 2016 combined with a slowing demand expansion. See e.g. Currie et al. 2015]

ARIMA model for $r^{12}\left(O_{t}^{U S \$}\right)$ (see Table 3). The output from this procedure can be interpreted as an univariate forecast for the annual change in Brent price, conditional to the corresponding end values ${ }^{11}$. This forecast is then feed to the transfer function in equations (6)-(7) to compute the corresponding inflation forecast.

The results of this exercise are summarized in Table 5 . As it can be seen, none of the scenarios considered yields a negative inflation forecast.

Table 5: Annual inflation rates $r^{12}\left(P_{t}^{E A}\right)$ corresponding to different scenarios for changes in brent prices $r^{12}\left(O_{t}^{U S \$}\right)$ in dollars.

\begin{tabular}{r|cc|cc|cc}
\hline & \multicolumn{3}{|c}{ Stable } & \multicolumn{2}{c}{ Pessimistic } & \multicolumn{2}{c}{ Extreme } \\
Year-Month & $r^{12}\left(P_{t}^{E A}\right)$ & $r^{12}\left(O_{t}^{U S \$}\right)$ & $r^{12}\left(P_{t}^{E A}\right)$ & $r^{12}\left(O_{t}^{U S \$}\right)$ & $r^{12}\left(P_{t}^{E A}\right)$ & $r^{12}\left(O_{t}^{U S \$}\right)$ \\
\hline $2015-12-01$ & 0.23 & -39.09 & 0.23 & -39.09 & 0.23 & -39.09 \\
$2016-01-01$ & 0.47 & -28.54 & 0.46 & -30.06 & 0.45 & -31.97 \\
$2016-02-01$ & 0.36 & -30.71 & 0.33 & -33.96 & 0.3 & -38.02 \\
$2016-03-01$ & 0.35 & -27.99 & 0.3 & -32.82 & 0.25 & -38.86 \\
$2016-04-01$ & 0.33 & -28.1 & 0.27 & -34.72 & 0.2 & -43.01 \\
$2016-05-01$ & 0.23 & -28.54 & 0.15 & -37.03 & 0.05 & -47.66 \\
$2016-06-01$ & 0.29 & -25.84 & 0.2 & -36.16 & 0.07 & -49.08 \\
$2016-07-01$ & 0.34 & -19.61 & 0.22 & -31.75 & 0.08 & -46.96 \\
$2016-08-01$ & 0.45 & -10.41 & 0.31 & -24.43 & 0.14 & -41.98 \\
$2016-09-01$ & 0.6 & -8.67 & 0.44 & -24.53 & 0.25 & -44.38 \\
$2016-10-01$ & 0.55 & -7.56 & 0.38 & -25.05 & 0.17 & -46.96 \\
$2016-11-01$ & 0.59 & -3.82 & 0.4 & -23.03 & 0.16 & -47.08 \\
$2016-12-01$ & 0.67 & 0 & 0.46 & -21 & 0.2 & -47.3 \\
\hline
\end{tabular}

These results suggest that the effect of oil prices on inflation is relevant but limited in the short term, as it is not enough by itself to create a long period of deflation. A deflationary spiral may occur however if an anemic inflation affects the agents' expectations and, through them, consumer decisions and economic activity. In this case, the short-term effect deflationary effects of oil prices would affect all the components of consumer prices.

\footnotetext{
${ }^{11}$ This procedure to compute the highest probability path for the exogenous input is a modest theoretical contribution of the paper.
} 


\subsection{Estimating the short-term effect of changes in oil prices}

We will use the transfer function (6)-(7) to decompose the inflation rate history in two additive components, one driven by the model input (changes in the oil prices) and another one driven by the model errors, being the former an approximation for the short-term effect of changes in oil prices on inflation.

In this case, we can compute the part of annual inflation that can be attributed to changes in Brent prices by propagating the following expression throughout the sample:

$$
r^{12}\left(\hat{P}_{t}^{O}\right)=0.0056 r^{12}\left(O_{t}^{U S \$}\right)+0.0046 r^{12}\left(O_{t-1}^{U S \$}\right) \quad t=2, \ldots, n
$$

Note that Expression 8 results immediately from the transfer function of equation $6^{12}$ On the other hand, the part of annual inflation corresponding to any other factors is trivially computed as:

$$
r^{12}\left(\hat{P}_{t}^{\text {Other }}\right)=r^{12}\left(P_{t}^{E A}\right)-r^{12}\left(\hat{P}_{t}^{O}\right)
$$

Figure 5 shows the profile of inflation in the E.A. versus the estimated effect of changes in Brent price, computed according to expression (8). It shows clearly that: (a) Brent prices have been a relevant factor to explain changes in consumer prices in the euro are ${ }^{13}$ and (b) from 2013 onwards their effect has been either neutral or deflationary.

Figure 6 provides further details on the effect of Brent prices from January 2014 to December 2016. It shows that they have been an important deflationary factor during this period, while the effect of other factors remained stable until November 2014, declined in December 2014 and January 2015, and started an inflationary cycle from February 2015 to May 2015.

\subsection{A proposal to track inflation/deflation risks in real- time}

The previous analysis suggests that, while oil prices are an important factor to explain recent deflationary pressures, a prolonged deflationary period would only occur if the negative evolution of crude factors creates a contagion on the other determinants of prices, e.g. through the agents expectations.

This idea suggests that the factor $r^{12}\left(\hat{P}_{t}^{\text {Other }}\right)$ could be used to track in realtime the risks of deflation. In particular, as most analysts predict that oil prices will continue their decline, the future behavior of this component will determine if inflation will stay in positive values or fall into a negative spiral.

This method is flexible so that, if other risk factors are identified (e.g., weak economic growth), they could be added as additional inputs to the transfer functions and taken into account accordingly.

\footnotetext{
${ }^{12}$ Casals et al. 2010] derive a procedure to compute this decomposition for a general transfer function.

${ }^{13}$ During the period analyzed, this factor accounted for $25 \%$ of the variance of the stationary transform of inflation.
} 


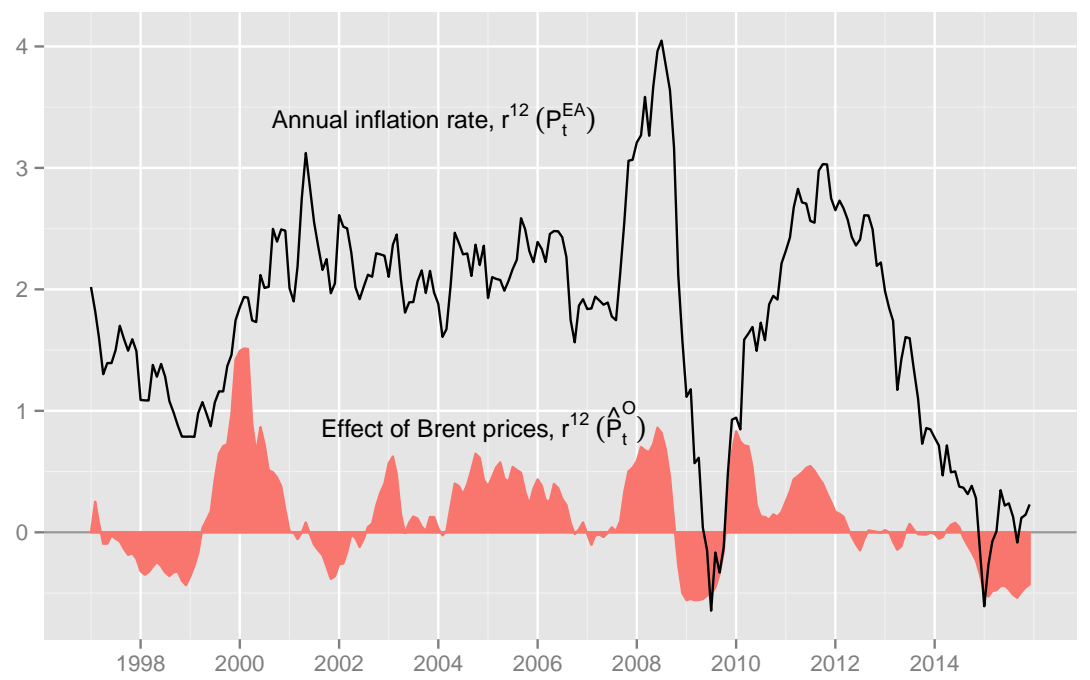

Figure 5: Annual inflation rates in the E.A. vs. the estimated effect of change in Brent prices.

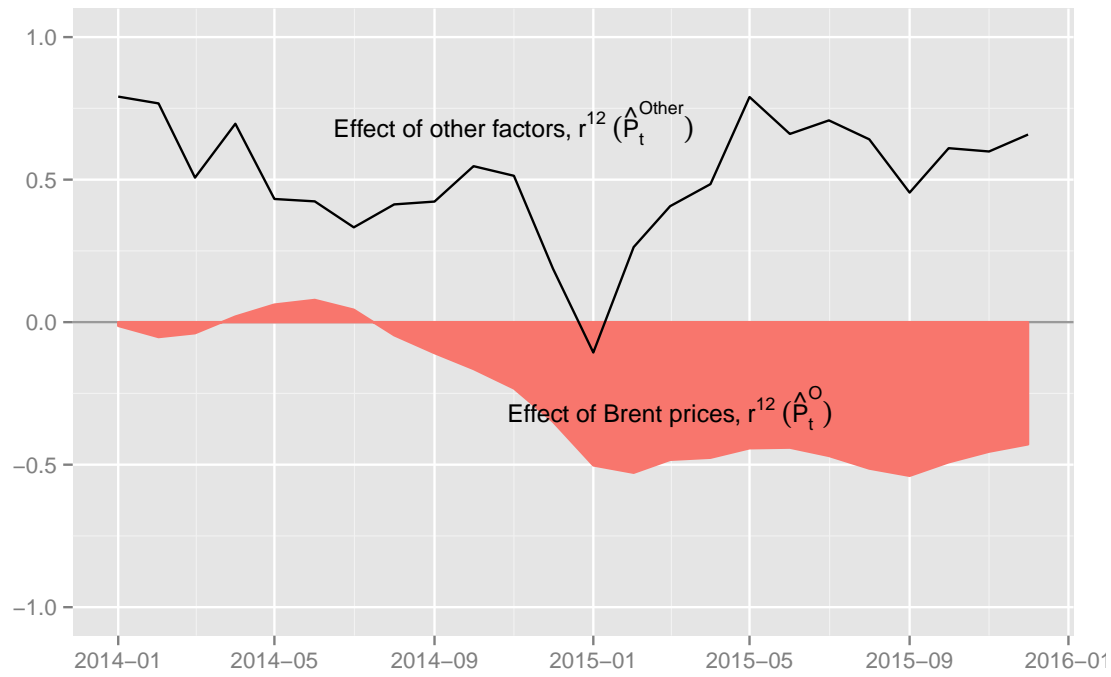

Figure 6: Components of inflation: effect of Brent prices vs. effects of other factors. 


\section{Conclusions}

The effect of oil price changes on inflation rates has received renewed interest. Contrary to the shocks in the 1970s, nowadays it is the deflationary effect of oil prices which is under scrutiny.

In this work, we propose a method to evaluate the effect of oil price changes on inflation, as well as an indicator of inflation adjusted for the short-term effect of oil prices. Tracking such an indicator may be an effective way to assess the risk of deflation in real time.

We apply the methodology to compute twelve-months ahead forecast for inflation in the E.A., conditional to different scenarios of oil price deflation and to estimate which part of the recent evolution of consumer prices can be attributed to changes in oil prices.

Our main findings are: (a) negative inflation is not expected for the twelvemonths-ahead forecasts in any of the three scenarios, (b) the short-time effect of oil on consumer prices is important, as it accounts for $25 \%$ of the variance of changes in inflation so, (c) a spiral of deflation/economic contraction could finally happen if a long period of anemic inflation/deflation affects the consumer expectations and, through them, the economic activity.

Future research will apply this framework to a disaggregate level of analysis. For example, the risk of deflation for specific countries may be evaluated. In addition, we will explore the effect of oil price variation on some specific components of the inflation rate.

Note that the methods proposed here could be applied to solve similar assessment and tracking needs in other frameworks, where a relevant economic magnitude, e.g., GDP, or unemployment, is affected by a driver variable such as a business climate indicator or some interest rates. In future papers we plan to explore some of these analyses.

All the calculations have been implemented in $E^{4}$, a free MATLAB toolbox for time series modeling, which can be downloaded at www.ucm.es/info/icae/e4. This website provides the source code for all the functions in the toolbox under the terms of the GNU General Public License, as well as a complete user manual and other reference materials. Besides $E^{4}$ we also used $\mathrm{R}$ and Gretl.

\section{References}

Ron Alquist, Lutz Kilian, and Robert Vigfusson. Forecasting the price of oil. In Graham Elliot and Allan Timmermann, editors, Handbook of Economic Forecasting. North-Holland, Amsterdam, 2013.

Luis Álvarez, Samuel Hurtado, Isabel Sánchez, and Carlos Thomas. The impact of oil price changes on spanish and euro area consumer price inflation. Economic Modelling, 28:422-431, 2011.

Brian D.O. Anderson and John B. Moore. Optimal Filtering. Prentice Hall, Englewood Cliffs (N.J.), 1979. 
Robert Barsky and Lutz Kilian. Oil and the macroeconomy since the 1970s. Journal of Economic Perspectives, 18:115-134, 2004.

Olivier Blanchard and Jordi Galí. The macroeconomic effects of oil shock: Why are the 2000s so different from the 1970s? In Jordi Galí and Mark Gertler, editors, International Dimensions of Monetary Policy, pages 373421. University of Chicago Press, Chicago, IL, 2010.

G E P Box, G M Jenkins, and G Reinsel. Time Series Analisys, Forecasting and Control. Prentice Hall, Englewood Cliffs, 3 edition, 1994.

J. Casals, M. Jerez, and S. Sotoca. Decomposition of a state-space model with inputs. Journal of Statistical Computation and Simulation, 80(9):979-992, 2010 .

Jeffrey Currie, Damien Courvalin, Abhisek Banerjee, Raquel Ohana, and Michael Hinds. Lower for even longer. Goldman Sachs, September, 2015.

J. DeGregorio, J. Landerretche, C. Neilson, C. Broda, and R. Rigobon. Another pass-through bites the dust? oil prices and inflation. Economia, 7(2):155-208, 2007.

Max Gillman and Anton Nakov. Monetary effects on nominal oil prices. North American Journal of Economics and Finance, 20:239-254, 2009.

C. W. J. Granger. Investigating causal relations by econometric models and cross-spectral methods. Econometrica, 37(3):424-438, 1969.

James Hamilton. Oil and the macroeconomy since world war ii. Journal of Political Economy, 91(2):228-248, 1983.

James Hamilton. Understanding crude oil prices. Energy Journal, 30(2):179206, 2009.

Mark Hooker. What happened to the oil price-macroeconomy relationship? Journal of Monetary Economics, 38(2):195-213, 1996.

Mark Hooker. Are oil shocks inflationary? asymmetric and nonlinear specifications versus changes in regime. Journal of Money, Credit and Banking, 34 (2):540-561, 2002.

Rebeca Jiménez-Rodríguez and Marcelo Sánchez. Oil price shocks and real gdp growth: empirical evidence for some oecd countries. Applied Economics, 37 (2):201-228, 2005.

Lutz Kilian. Exogenous oil supply shocks: How big are they and how much do they matter for the u.s. eonomy? Review of Economics and Statistics, 90(2): 216-240, 2008a. 
Lutz Kilian. A comparison of the effects of exogenous oil supply shocks on output and inflation in the $\mathrm{g} 7$ countries. Journal of the European Economic Association, 6(March):78-121, 2008b.

Lutz Kilian. The economic effects of energy price shocks. Journal of Economic Literature, 46(4):871-909, 2008c.

Lutz Kilian. Not all oil price shocks are alike: Disentangling demand and supply shocks in the crude oil market. American Economic Review, 99(3):1053-1069, 2009 .

Lutz Kilian. Oil price shocks: Causes and consequences. Annual Review of Resource Economics, 6:133-154, 2014.

Lutz Kilian and Clara Vega. Do energy prices respond to us macroeconomics news? a test of the hypothesis of predetermined energy prices. The Review of Economics and Statistics, 93(2):660-671, 2011. 


\section{Appendix}

We consider subsample estimations due to the relatively calm shown in first years for oil prices, the peak (in 2008) and collapse (in 2009), related with the 2008-2009 financial crisis, or the sharp decrease in 2014. Therefore we revise carefully the residuals of the transfer function models and we found long streaks of negative residuals in November 2008, May 2009 and July 2009, which profile suggested the step-type intervention effect

$$
S_{t}^{T}=\left\{\begin{array}{l}
1, \text { if } t \geq T \\
0, \text { otherwise }
\end{array}\right.
$$

, leading to the inclusion of three intervention effects: $S_{t}^{2008 / 11}, S_{t}^{2009 / 05}$ and $S_{t}^{2009 / 07}$.

Models (1A)-(2A), (4A)-(5A) and (6A-7A) combine the correspond structure of the transfer function models (1)-(2), (4)-(5) and (6)-(7) in the paper with the intervention terms required to capture the aforementioned step-effects.

As the models show, the results are very robust to the inclusion of the dummy variables, with the rest of the coefficients being very stable so that the prediction of inflation is similar. Nevertheless, according to the Bayesian Information Criteria, the model without the dummies is preferred (-95.49 without intervening and -95.06 afterwards).

- Models 1A and 2A. HICP in the Euro Area against Brent price in euros

$$
\begin{gathered}
\left.r^{12}\left(P_{t}^{E A}\right)=\underset{\left(8 \times 10^{-4}\right)}{0.0052}+\underset{\left(8 \times 10^{-4}\right)}{0.0042} L\right) r^{12}\left(O_{t}^{E U R}\right) \\
\left.-\underset{(0.161)}{(0.400}+\underset{(0.155)}{0.286 L}+\underset{(0.155)}{0.471} L^{2}\right) S_{t}^{2008 / 11} \\
-\underset{(0.155)}{0.362} S_{t}^{2009 / 05}-\underset{(0.153)}{0.391} S_{t}^{2009 / 07}+\hat{N}_{t}^{P} \\
\left(1-\underset{(0.068)}{0.118 L)} \nabla \hat{N}_{t}^{P}=\underset{(0.065)}{(1-0.466} L^{12}\right) \hat{a}_{t}^{P} \\
\hat{\sigma}_{P}=0.174 \quad \log -\operatorname{lik}=72.493
\end{gathered}
$$

- Models 4A and 5A. HICP in the Euro Area against Brent price in US\$ and the eur/US\$ exchange rate

$$
\begin{aligned}
& r^{12}\left(P_{t}^{E A}\right)=\left(\underset{\left(9 \times 10^{-4}\right)}{0.0057}+\underset{\left(9 \times 10^{-4}\right)}{0.0045} L\right) r^{12}\left(O_{t}^{U S \$}\right) \\
& +\underset{(0.0043)}{(0.0025}+\underset{(0.0043)}{0.0028 L) r^{12}}\left(E R_{t}\right) \\
& \left.-\underset{(0.161)}{0.441}+\underset{(0.157)}{0.308} L+\underset{(0.155)}{0.465} L^{2}\right) S_{t}^{2008 / 11} \\
& -\underset{(0.154)}{0.348} S_{t}^{2009 / 05}-\underset{(0.153)}{0.388} S_{t}^{2009 / 07}+\hat{N}_{t}^{P}
\end{aligned}
$$




$$
\begin{gathered}
(1-\underset{(0.068)}{0.114} L) \nabla \hat{N}_{t}=\left(\underset{(0.063)}{-0.454} L^{12}\right) \hat{a}_{t} \\
\hat{\sigma}_{P}=0.171 \quad \log -\operatorname{lik}=75.508
\end{gathered}
$$

- Models 6A and 7A. HICP in the Euro Area against Brent price in US\$

$$
\begin{gathered}
\left.r^{12}\left(P_{t}^{E A}\right)=\underset{\left(9 \times 10^{-4}\right)}{(0.0056}+\underset{\left(9 \times 10^{-4}\right)}{0.0043} L\right) r^{12}\left(O_{t}^{U S \$}\right) \\
\left.-\underset{(0.158)}{(0.413}+\underset{(0.154)}{0.297} L+\underset{(0.153)}{0.470 L^{2}}\right) S_{t}^{2008 / 11} \\
-\underset{(0.154)}{0.346} S_{t}^{2009 / 05}-\underset{(0.152)}{0.390} S_{t}^{2009 / 07}+\hat{N}_{t}^{P} \\
\left(1-\underset{(0.067)}{0.119 L)} \nabla \hat{N}_{t}^{P}=\underset{(0.063)}{\left(1-0.461 L^{12}\right)} \hat{a}_{t}^{P}\right. \\
\hat{\sigma}_{P}=0.172 \quad \log -\operatorname{lik}=75.005
\end{gathered}
$$

